\title{
Interactions of Binuclear Ruthenium(II) Complexes with Oligonucleotides in Hydrogel Matrix: Enantioselective Threading Intercalation into GC Context
}

\author{
Piotr Hanczyc ${ }^{1,2}$, Per Lincoln ${ }^{1}$, Bengt Norden ${ }^{1 *}$ \\ ${ }^{1}$ Department of Chemical and Biological Engineering, Chalmers University of Technology, SE- \\ 41296 Gothenburg, Sweden \\ ${ }^{2}$ Institute of Physical and Theoretical Chemistry, Wroclaw University of Technology, 50-370, \\ Wroclaw, Poland \\ *corresponding author: norden@chalmers.se \\ Abstract
}

A stretched poly-(vinyl alcohol) (PVA) film provides a unique matrix that enables also short DNA oligonucleotide duplex to be oriented and studied by linear dichroism (LD). This matrix further allows controlling DNA secondary structure by proper hydration (A or B form), and such humid films could potentially also mimic the molecular crowding in cellular contexts. However, early attempts to study intercalators and groove binders for probing DNA in PVA failed due to competitive matrix binding. Here we report the successful orientation in PVA of DNA oligonucleotide duplex hairpins with thread-intercalated binuclear complex $\left[\mu-\left(11,11^{\prime}-\right.\right.$ bidppz)(phen $\left.)_{4} \mathrm{Ru}_{2}\right]^{4+}$, and how $\mathrm{LD}$ depends on oligonucleotide sequence and metal center chirality. Opposite enantiomers of the ruthenium complex, $\Delta \Delta$ and $\Lambda \Lambda$, were investigated with respect to enantioselectivity towards GC stretches as long as $22 \mathrm{bp}$. LD, supported by emission kinetics, reveals that threading intercalation occurs only with $\Delta \Delta$ whereas $\Lambda \Lambda$ remains externally 
bound, probably in either or both of the grooves of the GC-DNA. Enantioselective binding properties of sterically rigid DNA probes such as the ruthenium complexes could find applications for targeting nucleic acids, e.g., to inhibit transcription in therapeutic context such as treatment of malaria or cancer.

Keywords: synthetic DNA, PVA-DNA interactions, threading intercalation, DNA recognition, molecular crowding

\section{Introduction}

DNA short oligonucleotides of defined sequence are becoming widely used in studies on DNAdrug interactions ${ }^{1-5}$. Highly specific and enantioselective DNA-binding transition metal complexes constitute an interesting class of drugs exhibiting versatile properties that, due to the rigid coordination framework with possibilities to vary the three-dimensional structure could be tuned for specific tasks with respect to DNA structure and sequence composition ${ }^{6-9}$. Ruthenium (II) complexes, in particular those comprising the dipyrido[3,2-a:2', $\left.3^{\prime}-c\right]$ phenazine (dppz) ligand and derivatives thereof, have been intensively studied due to their environmental sensitive luminescence which make them versatile spectroscopic probes for DNA ${ }^{10,11}$. For example, upon intercalation into DNA, the complexes $\left[\mathrm{Ru}(\mathrm{bpy})_{2} \mathrm{dppz}\right]^{2+}$ show an almost three order of magnitude increase in luminescence intensity (the light-switch effect) upon intercalation into $\mathrm{DNA}^{12-16}$. Two monomeric $\left[\mathrm{Ru}(\mathrm{phen})_{2} \mathrm{dppz}\right]^{2+}$ moieties connected by a single bond between the dppz moieties makes the resulting dimeric complex bulky and relatively rigid. Therefore, a 
substantial thermal opening of the DNA double helix is required to allow one of the ruthenium centers to pass through the DNA base stack and place one of the dppz bridging moieties into an intercalation position ${ }^{17-19}$. This unusual threading intercalation mode of binding shows a unprecedented sensitivity of the association and dissociation kinetics to the sequence of the DNA, and such binuclear complexes appear to be promising candidates, for example, for developing therapeutics for cancer treatment ${ }^{20-22}$ and in contexts of other diseases requiring high nucleic acid selectivity ${ }^{23-25}$. In addition, intramolecular rigidity improves multiphoton properties and thus potential applications of these compounds are also found in multiphoton microscopy or photodynamic therapy (PDT) ${ }^{26}$.

Incorporating oligonucleotides with a bound drug into poly (vinyl alcohol) matrix opens a wide spectrum of research opportunities. Stretched humid PVA allows study of macroscopically anisotropic systems by linear dichroism, a method that has been traditionally confined to long DNA ( $>500$ base pairs), that may be aligned by shear flow, and therefore not allowed systematic variations of uniform binding site sequence. However, today short DNA duplexes of defined sequence down to $20 \mathrm{bp}{ }^{27}$ may be easily aligned in PVA matrix, another advantage of which is also the controllable hydration allowing manipulating the secondary structure of DNA with possibility for switching between A- and B-forms ${ }^{28}$. A great disadvantage, however, of the PVA system is the low dielectric constant of the medium surrounding the DNA, which will compete with the hydrophobic interactions of the interior of DNA and thus with the binding of added probe molecules. Thus, the interactions of traditional intercalators (ethidium) and minor-groove binders (Hoechst) cannot be studied because these drugs to a great extent prefer the PVA environment where they become aligned parallel with the polymer chains. By contrast, the 
strongly binding and slowly dissociating binuclear ruthenium complexes may be studied in PVA as they will remain bound to the DNA despite the decreased dielectric constant of the surrounding. Using the advantage of strong binding to DNA of binuclear complexes we use the PVA system to better understand the molecular recognition properties of specific hairpin DNA sequences.

Here we report on interactions of short oligonucleotide HEG-linked hairpins composed of either alternating $\mathrm{AT}$ or GC sequences with either of the two enantiomers $\Delta \Delta$ and $\Lambda \Lambda$ of the binuclear complex $\left[\left(11,11^{\prime} \text {-bidppz }\right)(\text { phen })_{4} \mathrm{Ru}_{2}\right]^{4+}(\mathrm{Fig}$ 1). Strong electrostatic interaction suppresses dissociation of the complex once it is threaded between DNA bases. The tetravalent charge is a prerequisite when DNA-drug interactions are studied in poly (vinyl) alcohol where binding equilibrium is effected by the polymeric environment and DNA-drug complexes are generally destabilized as an effect of low dielectric constant of the surrounding, decreasing the hydrophobic contribution to binding energy ${ }^{29}$. As potential lead compounds for development of genetic drugs it is essential to study the interactions with specific short sequences of DNA. Earlier reports have mostly focused on interactions with AT polymers because of their strong luminescence properties and interactions with GC sequences have been less studied due to the generally low quantum yields for binuclear complexes in poly $(\mathrm{dGdC})_{2}{ }^{19}$. Here we are able to study interactions of both of the enantiomers $\Delta \Delta$ and $\Lambda \Lambda$ with both AT and GC oligomers using polarized light spectroscopy methods and emission kinetics. 
A

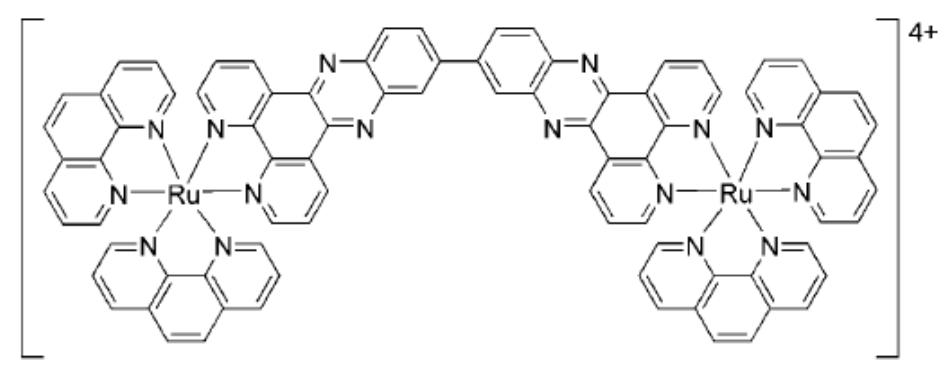

B

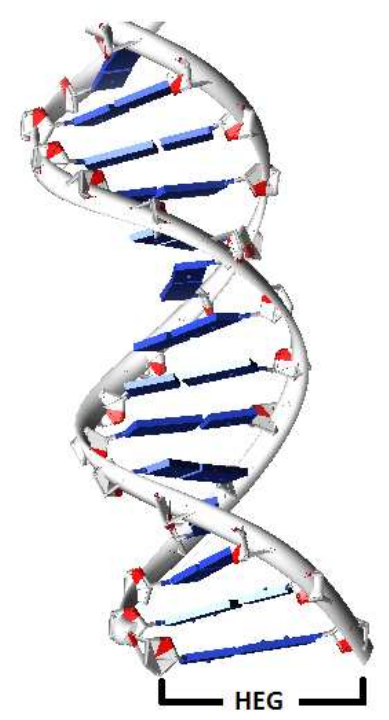

Fig. 1 A) $\left[\left(11,11^{\prime} \text {-bidppz }\right)(\text { phen })_{4} \mathrm{Ru}_{2}\right]^{4+}$, B) Functionalized hairpin AT and GC oligonucleotides with hexaethylene glycol (HEG)

Materials and methods

Materials. Poly(vinyl alcohol), PVA, with an average molecular weight of 80,000 was purchased from Du Pont (Sweden) under the commercial name Elvanol 71-30. HEG-linked oligonucleotides were obtained from ATDBio (UK), dissolved in $150 \mathrm{mM} \mathrm{NaCl}$ and used without further purification. Enantiomers of binuclear ruthenium complex [(11,11'bidppz)(phen) $\left.)_{4} \mathrm{Ru}_{2}\right]^{4+} \Delta \Delta$ and $\Lambda \Lambda$ were synthesized as previously reported ${ }^{18,19}$.

Film sample preparation. PVA powder $(10 \% \mathrm{w} / \mathrm{v})$ in a $2 \mathrm{mM} \mathrm{NaCl}$ solution was stirred for 5 min at room temperature, and then incubated at $80 \mathrm{C}^{\circ}$ under continued stirring until a transparent viscous solution was obtained (ca $30 \mathrm{~min}$ ). Samples of PVA, DNA and binuclear complexes 
were prepared by mixing the three constituents at volume ratios 100:20:1 using stock solutions with the following concentrations: $10 \% \mathrm{w} / \mathrm{v}(0,1 \mathrm{~g} / \mathrm{ml})$ PVA, $0,7 \mathrm{mM}$ (TA)16-HEG-(TA)16, and 0,8 mM CCGG(CG)7-HEG-(CG)7CCGG (HEG=hexaethylene glycol) and binuclear complex solution $0,45 \mathrm{mM}$ for $\Delta \Delta-\mathrm{Ru}$ and $0,49 \mathrm{mM}$ for $\Lambda \Lambda$-Ru. DNA- complex, prior mixing with PVA, was kept in $37 \mathrm{C}^{\circ}$ for $2 \mathrm{~h}$ in order to induce transient opening of DNA double helix for threading as described elsewhere ${ }^{17}$. Final samples composition were $0,083 \mathrm{~g} / \mathrm{ml}$ of PVA, (TA)16-HEG(TA)16 and CCGG(CG)7-HEG-(CG)7CCGG were between $115 \mu \mathrm{M}$ and $135 \mu \mathrm{M}$ and enantiomers concentration vary between $3,7 \mu \mathrm{M}$ and $4,1 \mu \mathrm{M}$. The final mixing ratios in all samples were then estimated ( $r=$ [complex]/[DNA base]) for AT and GC to be between $r=0.03$ and 0.035. The ionic strength of DNA-complex samples was $150 \mathrm{mM} \mathrm{NaCl}$ and then mixed with PVA dissolved in $2 \mathrm{mM} \mathrm{NaCl}$ from the start. The estimated final salt concentration in the film samples were approximately $25 \mathrm{mM} \mathrm{NaCl}$.

PVA films containing DNA-complex were prepared following the established procedures ${ }^{29}$. Briefly, the PVA-DNA-binuclear complex solution (typically $1 \mathrm{ml}$ ) was poured on a glass slide and allowed to evaporate in the air to form a dehydrated PVA sheet (2 days at room temperature in dust-free environment). The sheets were then assembled into a stretching device and hydrated to become humid films, inside a closed chamber (with silica windows allowing optical measurements) where relative humidity was controlled by the equilibrium water pressure above a salt solution in a container at the bottom of the chamber $(100 \%$ r.h. for water and $75 \%$ r.h. for saturated $\mathrm{NaCl}$ solution). The dry PVA sheet, which is brittle, takes up water rather quickly (30 minutes) to become rubber-elastic and easy to stretch without risk of rupture. 
Finally the hydrated films were stretched under the same controlled humidity conditions to a predetermined stretching ratio $R_{s}=D \| / D_{\perp}$ between length and width of stretched film $\left(R_{s}=1.5\right.$ if not otherwise stated).

$U V$-Vis Spectroscopy. Absorption spectra were recorded on a CARY-5000 spectrophotometer, using as a reference PVA films prepared as above but lacking the DNA.

Circular Dichroism. Circular Dichroism (CD) spectra were recorded on a Chirascan CD spectropolarimeter with a buffer as a baseline.

Linear Dichroism. Linear dichroism (LD) is defined as the difference in absorbance of light linearly polarized parallel $\left(\mathrm{A}_{\|}\right)$and perpendicular $\left(\mathrm{A}_{\perp}\right)$ to the macroscopic axis of orientation (in this study the stretching direction of the film).

$$
L D=A_{\|}-A_{\Perp}
$$

LD spectra of short DNA molecules oriented in such stretched films can provide information on the rate of orientation and structure of DNA while a non-stretched (isotropic) film should exhibit zero LD. LD spectra were recorded on a Chirascan CD spectrophotometer (Applied Photophysics) equipped with an LD accessory unit - a stretching device with the film sample inside a humidity chamber.

The reduced $\mathrm{LD}\left(\mathrm{LD}^{\mathrm{r}}\right)$ is obtained by dividing the $\mathrm{LD}$ by the absorbance of the corresponding isotropic sample $\left(\mathrm{A}_{\mathrm{iso}}\right)$, which for an anisotropic uniaxial sample can be obtained as $\mathrm{A}_{\mathrm{iso}}=\left(\mathrm{A}_{\|}+2 \mathrm{~A}_{\perp}\right) / 3^{28}$, so that 


$$
L D^{r}=\frac{L D}{A_{i s o}}=\frac{3 \cdot L D}{A_{\|}+2 A_{1}}
$$

For molecules with a uniaxial orientation distribution, as can be expected for DNA in PVA matrix since the films are thick (30 $\mu \mathrm{m}$ for dry film) compared to the helix diameter $(2 \mathrm{~nm}), \mathrm{LD}^{\mathrm{r}}$ is a product of an orientation factor $\mathrm{S}$ and an optical factor $\mathrm{O}$. The uniaxial distribution is not due to the DNA but to the flow properties of the PVA "melt". A cylindrical rod of DNA by macroscopic symmetry must have also a microscopic uniaxial distribution, but this is not obviously the case for a thin film. The issue is discussed in reference ${ }^{30}$ where it is also concluded from experiments in dry PVA that for small deformations the distribution is indeed uniaxial.

$$
L D^{r}=S \cdot O=\frac{3}{2} S\left(3 \cos ^{2} \alpha-1\right)
$$

The optical factor $O$ is related to the angle $\alpha$ between the local helix axis and the light absorbing transition moment of a DNA base. The orientation factor $S=1 / 2\left(3<\cos ^{2} \theta>-1\right)$ corresponds to the effective orientation of the DNA helix in the PVA film, where $\theta$ is the angle between the macroscopic stretching direction and the local helix axis of a particular molecule, and where the average runs over all parts of DNA molecules in the sample. $S=1$ for perfect orientation of helix axis parallel with stretch direction, i.e. $\theta=0$. The degree of helix orientation $S$ was calculated from the $\mathrm{LD}^{\mathrm{r}}$ values at $260 \mathrm{~nm}$ using the average angle $\alpha_{\mathrm{DNA}}=86^{\circ}$ for the DNA bases ${ }^{31}$. The binding angle $\alpha$ for a binuclear complex with respect to the DNA helix axis can then be calculated from the $\mathrm{LD}^{\mathrm{r}}$-value of dppz band at $\sim 320 \mathrm{~nm}$ which is polarized along the long-axis of the intercalating dppz moiety ${ }^{12}$. 
Emission kinetics. Emission kinetics was studied using Varian Cary Eclipse spectrofluorometer equipped with a multicell temperature controller. The samples were excited at $\lambda_{\mathrm{ex}}=410 \mathrm{~nm}$ and emission recorded at $\lambda_{\mathrm{em}}=650 \mathrm{~nm}$.

\section{Results}

Absorption spectroscopy. The absorption spectra of both $\Delta \Delta$ and $\Lambda \Lambda$ show a distinct decrease in absorbance (hypochromism) for both the intraligand $\pi \rightarrow \pi^{*}$ transitions of the bidppz ligand (at 310 and $410 \mathrm{~nm}$ ) as well as in the metal to ligand charge transfer (400-500 nm) region upon addition either AT or GC HEG-linked oligonucleotides [Fig. 2] evidencing the close interaction of the chromophores of the probe with the nucleobases. The hypochromicity is almost identical for both $\Delta \Delta$ and $\Lambda \Lambda$ in case of $\mathrm{AT}$ sequence though with a slight difference in absorption amplitude upon interaction with GC DNA. However, undoubtedly both enantiomers strongly interact also with GC sequence. Both samples were heated for $2 \mathrm{~h}$ in $37 \mathrm{C}^{\mathrm{o}}$. The change in absorbance is slightly larger in $\Delta \Delta$ than $\Lambda \Lambda$ but the shape in both cases is similar. Absorption experiments performed on isotropic as well as stretched PVA samples showed further amplitude decrease when films had been stretched [Fig S2]. However, neither hydration conditions nor stretching ratio seem to influence the spectral shapes. 
A

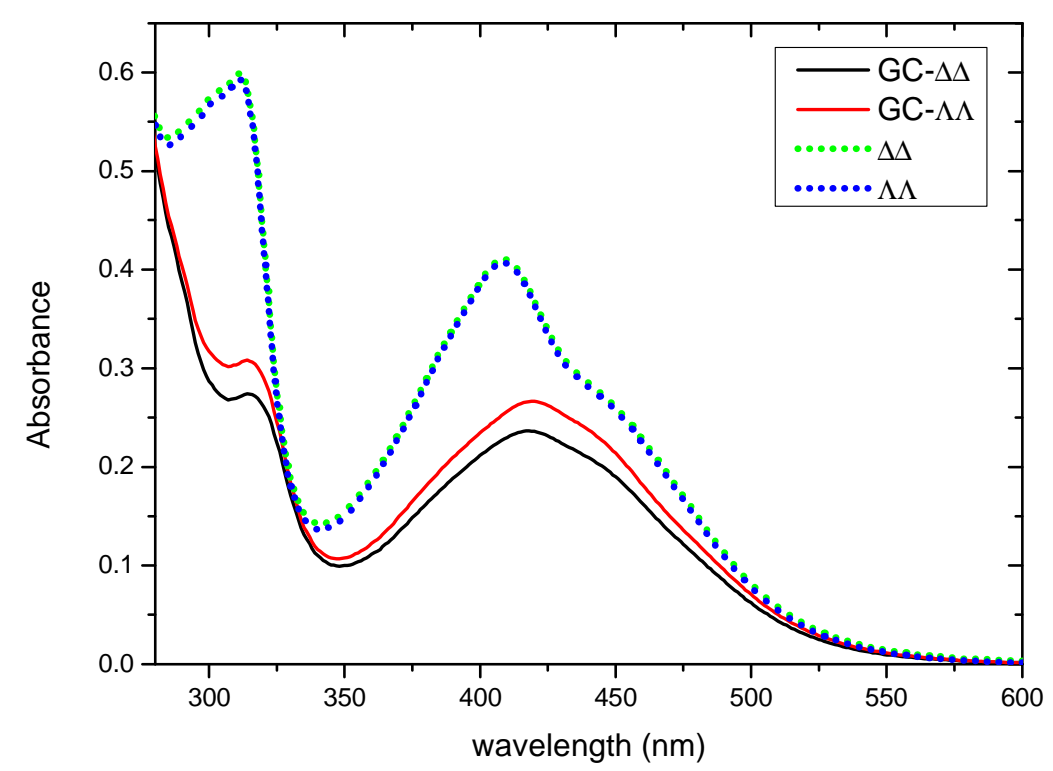

B

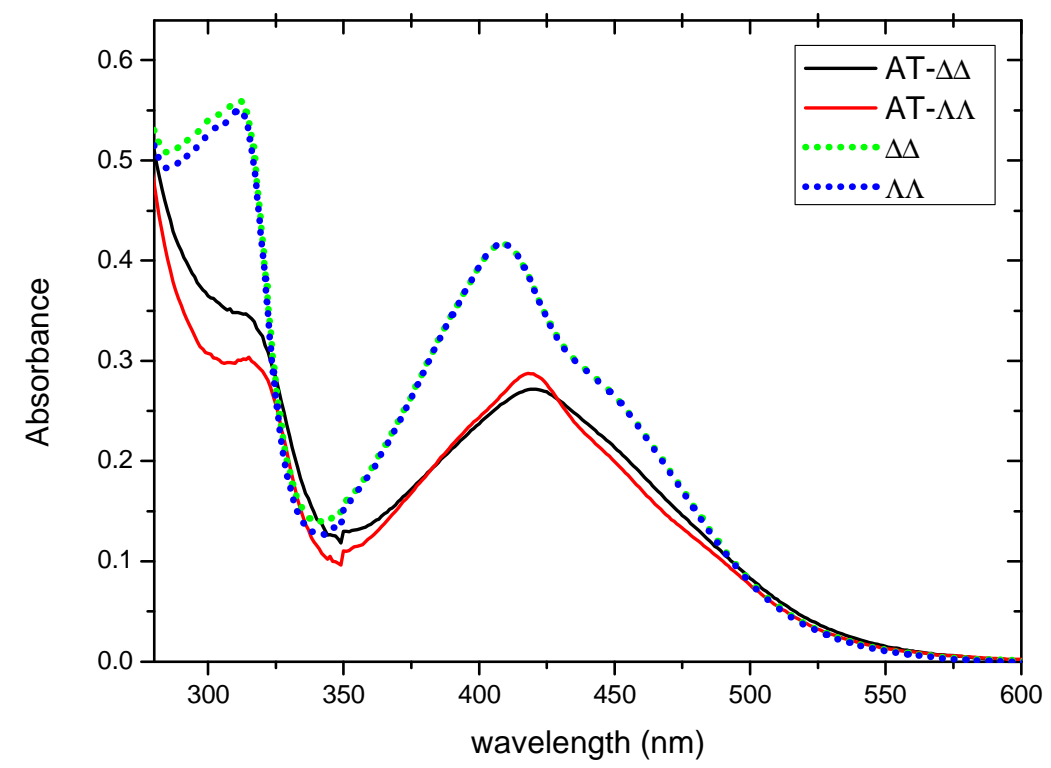

Fig. 2 Absorption spectra of $\Delta \Delta$ and $\Lambda \Lambda$ in $25 \mathrm{mM} \mathrm{NaCl}$ water solution (green and blue) and in the presence of A) GC (red and black) and B) AT oligonucleotide (red and black) after heating in $37 \mathrm{C}^{\circ}$ 
LD spectroscopy. Binuclear complexes with HEG-linked short oligonucleotides of either AT or GC sequences were mixed with $\Delta \Delta$ and $\Lambda \Lambda$ in ratio approximately $1: 1$. Further incorporation into PVA allows for aligning the DNA-drug complex and investigating the system by linear dichroism. Experiments with humidity control performed on the DNA-drug indicated improved stability of both AT and GC oligonucleotides in the PVA when in presence of the binuclear ruthenium complex [Fig. 3]. LD spectra of free HEG-linked AT and GC in PVA revealed large variations with humidity conditions but in contrast to pure oligonucleotide duplexes (without HEG) ${ }^{27}$ the helical structure was retained in the dehydrated state at $75 \%$ r.h. conditions even for as short DNA as 22 bp GC [Fig. S1]. Although the intraligand $\pi \rightarrow \pi^{*}$ bands are covering the LD from DNA transition dipole moments the difference in LD magnitude between $75 \%$ r.h. and $100 \%$ r.h. is relatively small when comparing the LD of free DNA with that of the DNA-drug complex.

Analysis of LD spectra confirmed threading intercalation of both $\Delta \Delta$ and $\Lambda \Lambda$ into HEG-linked AT sequence. Strong negative peaks at around $320 \mathrm{~nm}$ which originates from a $\pi \rightarrow \pi^{*}$ transition polarized parallel to long axis of bidppz ligand, which is expected responsible for intercalation, clearly shows the preferred perpendicular arrangement of this ligand with respect to the DNA helix orientation [Fig. $3 \mathrm{C}$, D]. From $\mathrm{LD}^{\mathrm{r}}$ spectra calculated by dividing LD with the isotropic absorption of the same film sample, interestingly, $\Delta \Delta$ is found to align more perpendicular to the helix axis in dehydrated state than the opposite enantiomer $\Lambda \Lambda$. At $100 \%$ r.h. condition the orientation of both enantiomers, however, becomes similar [Fig. 4 A]. With the GC oligonucleotide only the $\Delta \Delta$ complex exhibits a strong negative LD in the dppz region. In case of 
$\Lambda \Lambda$ it is negative at low water activity conditions but becomes more positive in $100 \%$ r.h. [Fig. 3 $\mathrm{A}, \mathrm{B}]$. Also the shape of the $\mathrm{LD}$ at the peak at $320 \mathrm{~nm}$ is changing with increasing water content in the film. There is a clear tendency to red shift, similar to the absorption spectrum of pure $\Lambda \Lambda$ complex in PVA, with increasing water content in the film indicating dissociation from the DNA. From a linear combination of the LD spectrum of free complex in PVA with the one in presence of DNA at $75 \%$ r.h. conditions, it is concluded that about $25 \%$ of the complex is dissociated and aligned instead parallel with the PVA polymer chains in high water content film [Fig. S3]. Fast dissociation is assigned to be a result of external binding mode on DNA, possibly groove binding. However, as an effect of dominant positive LD by interaction of the dissociated probe with the anisotropic PVA environment, it is difficult to estimate the LD of the remaining DNA-bound complex and thus the binding angle between DNA and $\Lambda \Lambda$ complex.

A

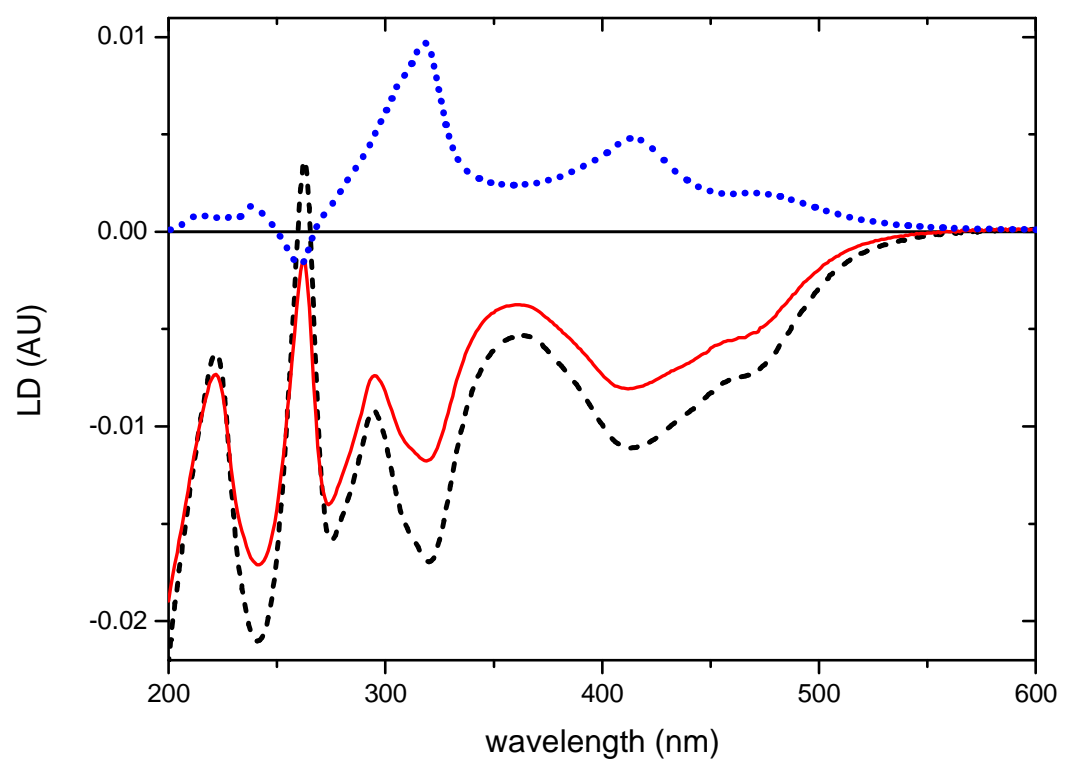


B

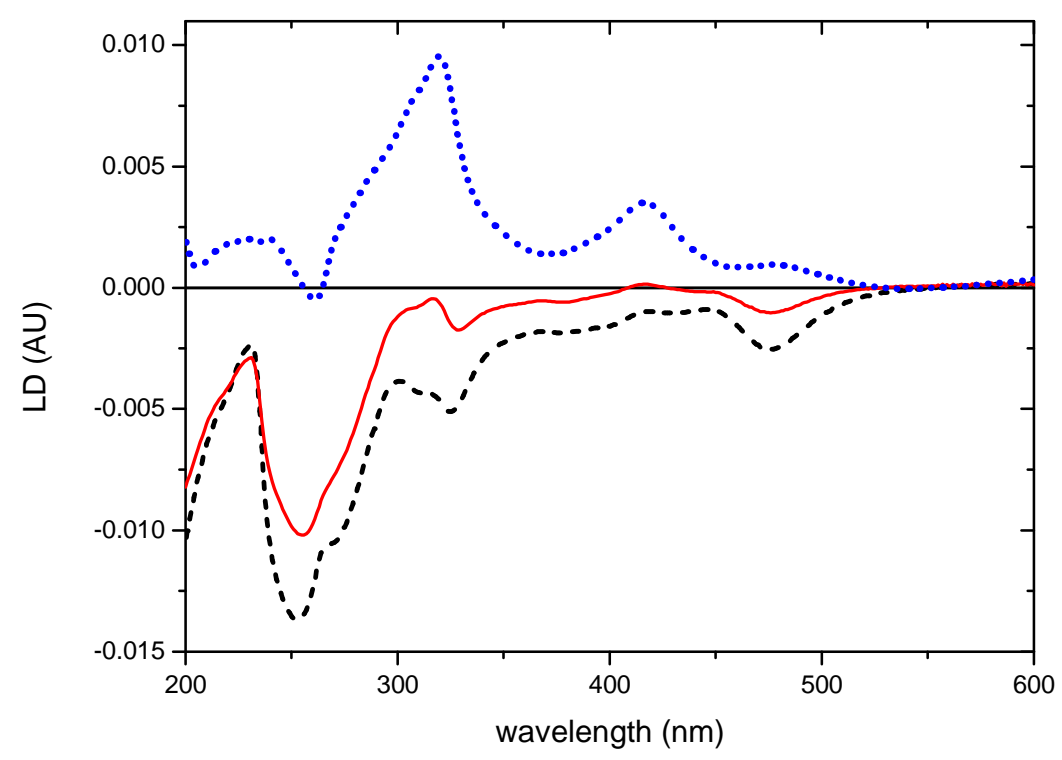

C

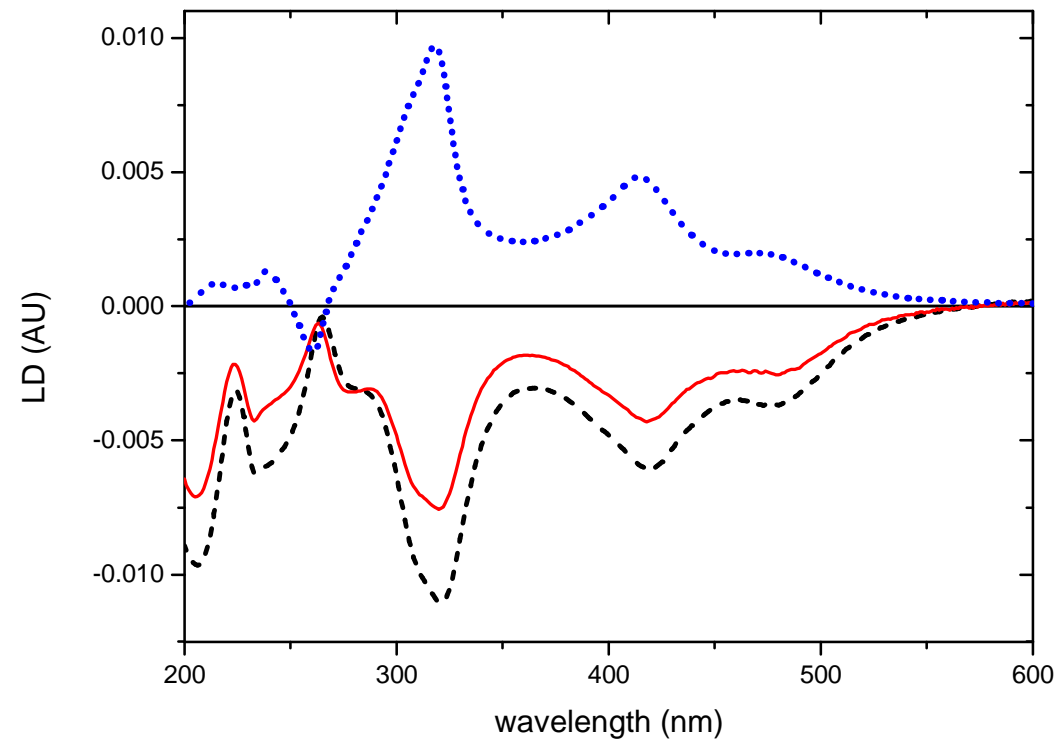


$\mathrm{D}$

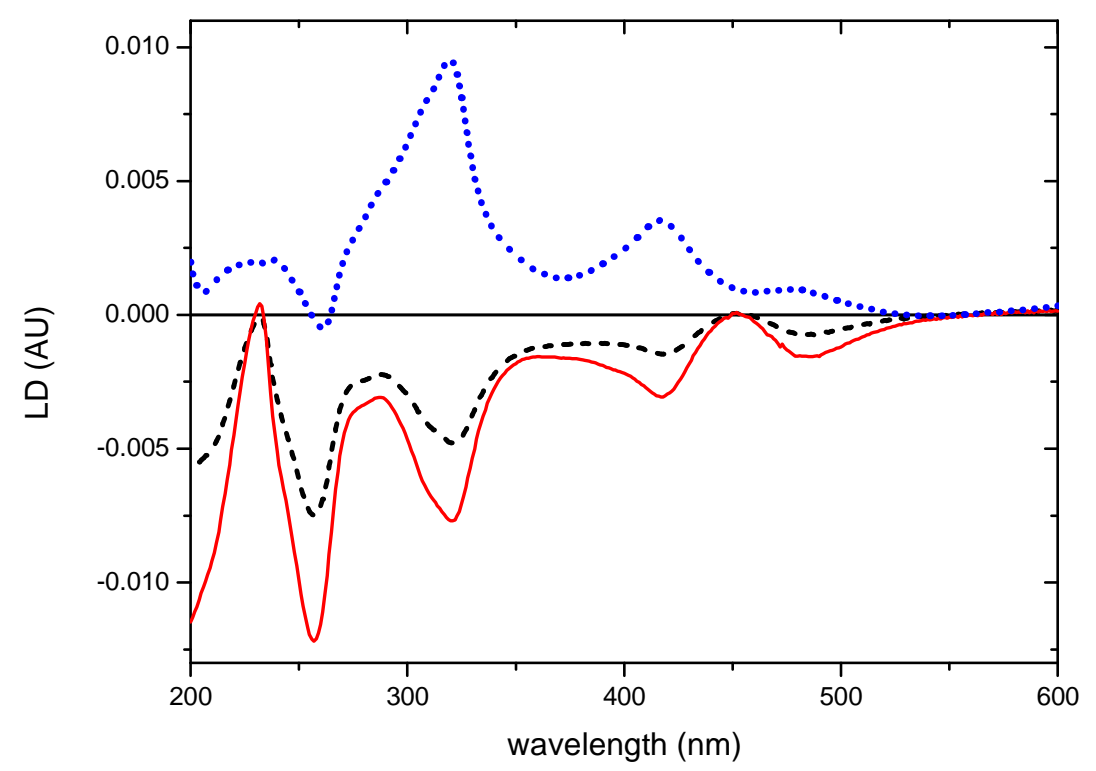

Fig. 3 Linear dichroism spectra of AT and GC oligonucleotides interacting with ruthenium(II) complexes in two states of PVA hydration level: film equilibrated in $100 \%$ relative humidity (r.h.) conditions and in $75 \%$ r.h., respectively, in the following order: 22 bp GC-DNA with A) $\Delta \Delta$ enantiomer in $100 \%$ r.h. (solid red) and in $75 \%$ r.h. (dashed black) , B) $\Lambda \Lambda$ enantiomer in $100 \%$ r.h. (solid red) and in $75 \%$ r.h. (dashed black) showing a change in LD shape between 100\% r.h. and 75\% r.h. due to dissociation and alignment of free complex in PVA and 32 bp AT-DNA with C) $\Delta \Delta$ enantiomer in 100\% r.h. (solid red) and in $75 \%$ r.h. (dashed black), D) $\Lambda \Lambda$ enantiomer in $100 \%$ r.h. (solid red) and $75 \%$ r.h. (dashed black). In all graphs free $\Delta \Delta$ and $\Lambda \Lambda$ complexes when oriented alone in PVA shown for comparison (within measuring errors identical spectra, blue dotted). Samples were in $25 \mathrm{mM} \mathrm{NaCl}$. 
A

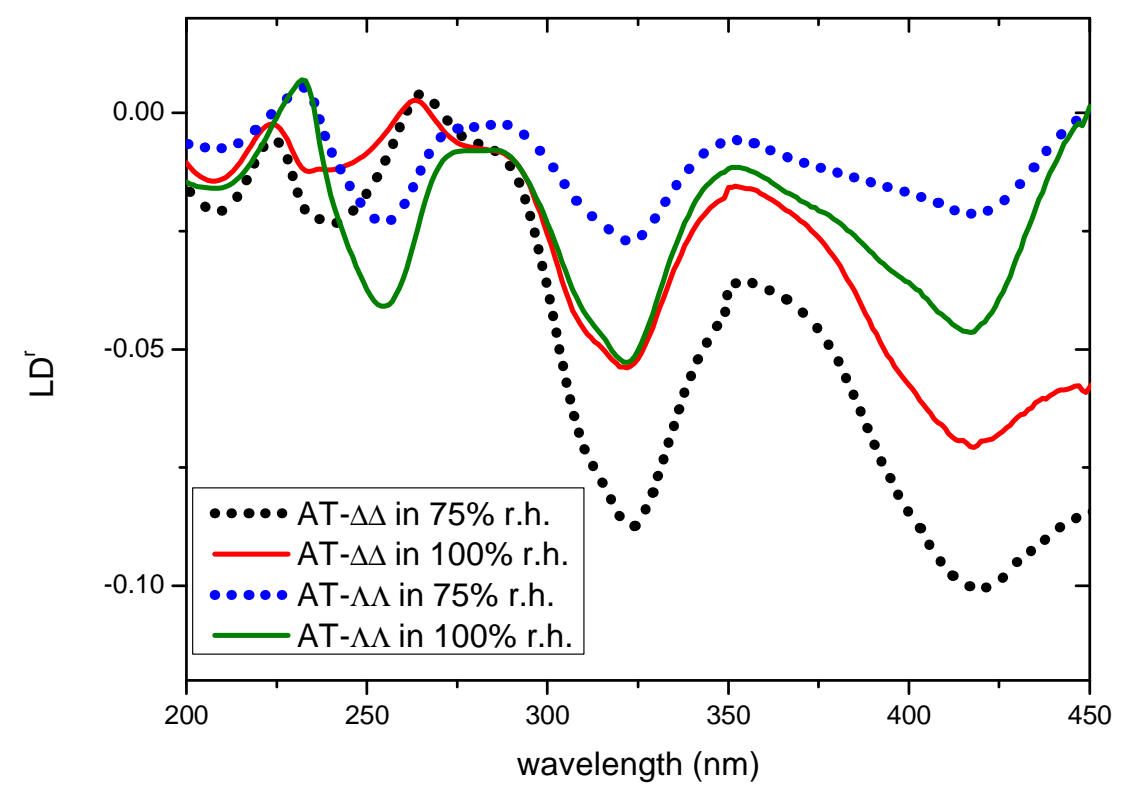

B

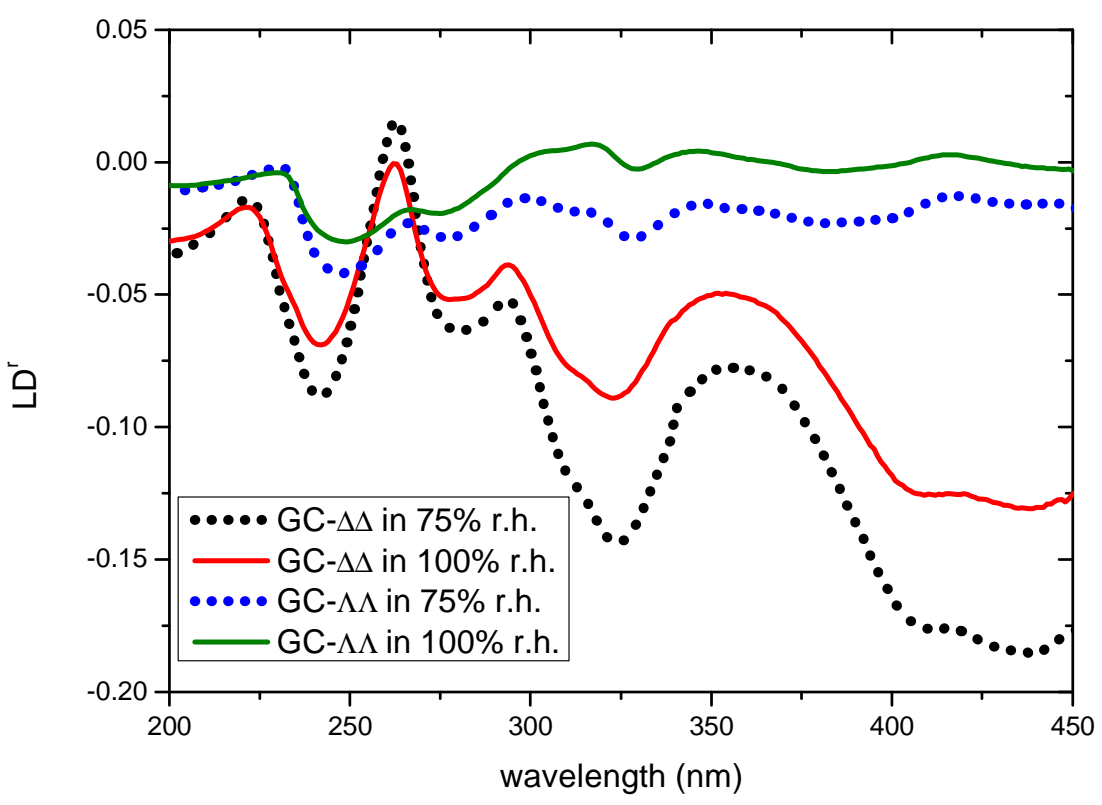


Fig. $4 \mathrm{LD}^{\mathrm{r}}$ curves of $\Delta \Delta$ and $\Lambda \Lambda$ in $75 \%$ r.h. (dotted) and $100 \%$ r.h. (solid) conditions with A) AT-DNA, B) GC-DNA

CD spectroscopy. In order to further confirm and characterize the phenomenon of threading intercalation into the AT and GC hairpin oligonucleotides, $\mathrm{CD}$ experiments in $25 \mathrm{mM} \mathrm{NaCl}$ solution were performed. The asymmetry of interactions will be displayed as "Perturbation CD", PCD, defined as the differential between the CD spectrum of pure enantiomer ruthenium complex and the spectrum when in presence of DNA. This differential spectrum represents in part an induced $\mathrm{CD}$, due to the chiral arrangement of nucleobases providing non-degenerate rotational strength in the absorption active transitions, in part it is due to change of the absorption intensity which correspondingly scales (symmetrically) the CD of the complex. Different shapes of the perturbation spectra of opposite enantiomers are expected: the induced $\mathrm{CD}$ will have the same sign provided the orientation of the chromophore relative to the DNA bases is the same, for example, positive for a transition moment aligned along one of the grooves ${ }^{32}$. However, hypochromism will decrease the $\mathrm{CD}$ of an enantiomer (thus with opposite signs for the two enantiomers). If the degree of interaction is different for the two enantiomers the latter mechanism will add further to the difference between the perturbation CD spectra.

First of all the $\mathrm{CD}$ of the pure enantiomers were measured and then either AT or GC oligomer added of the same oligonucleotide length as used in LD-PVA experiments and described above. Taking into consideration the LD results showing that $\Delta \Delta$ and $\Lambda \Lambda$ are threading AT-DNA also the PCD results indicate strong interactions with AT sequences in case of both enantiomers [Fig. 
5A]. Similar observations made by Westerlund et al. ${ }^{23}$ were interpreted in terms of threading intercalation in long poly $(\mathrm{dAdT})_{2}$.

However, with GC probably only $\Delta \Delta$ is thread-intercalated while $\Lambda \Lambda$ is possibly groove bound to DNA as judged by the much smaller PCD in the latter case [Fig. S4]. Binuclear complex $\Delta \Delta$ is significantly influencing the structure of HEG-linked GC oligonucleotide whereas $\Lambda \Lambda$ is not perturbing as much the DNA structure and the DNA bands in UV can be clearly distinguished [Fig. 5 B].

A

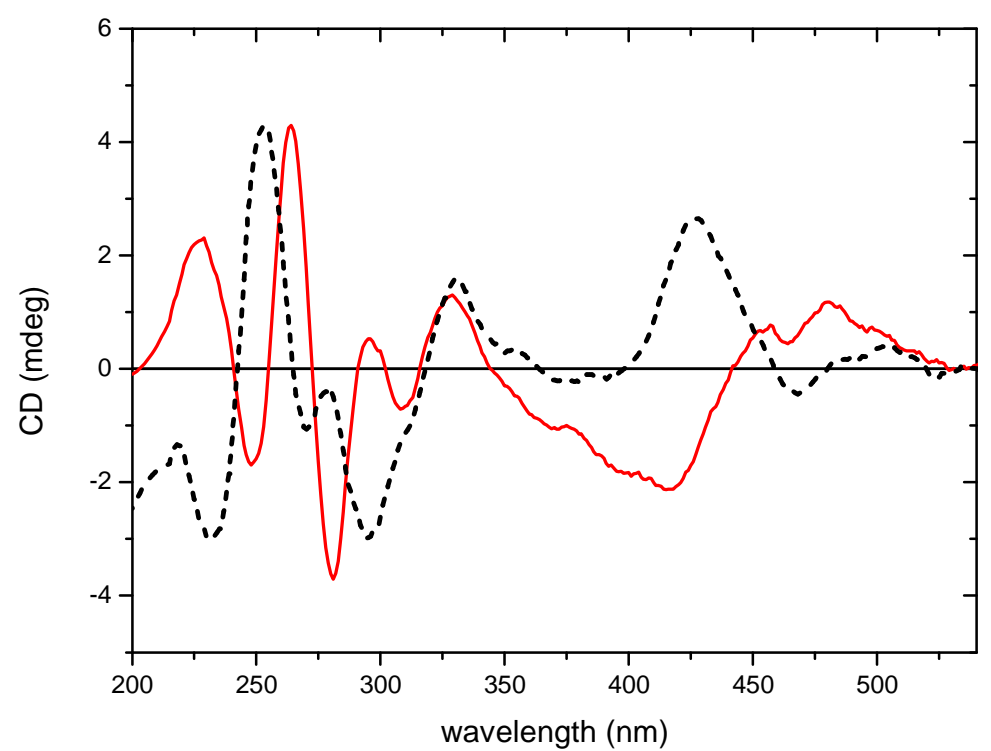


B

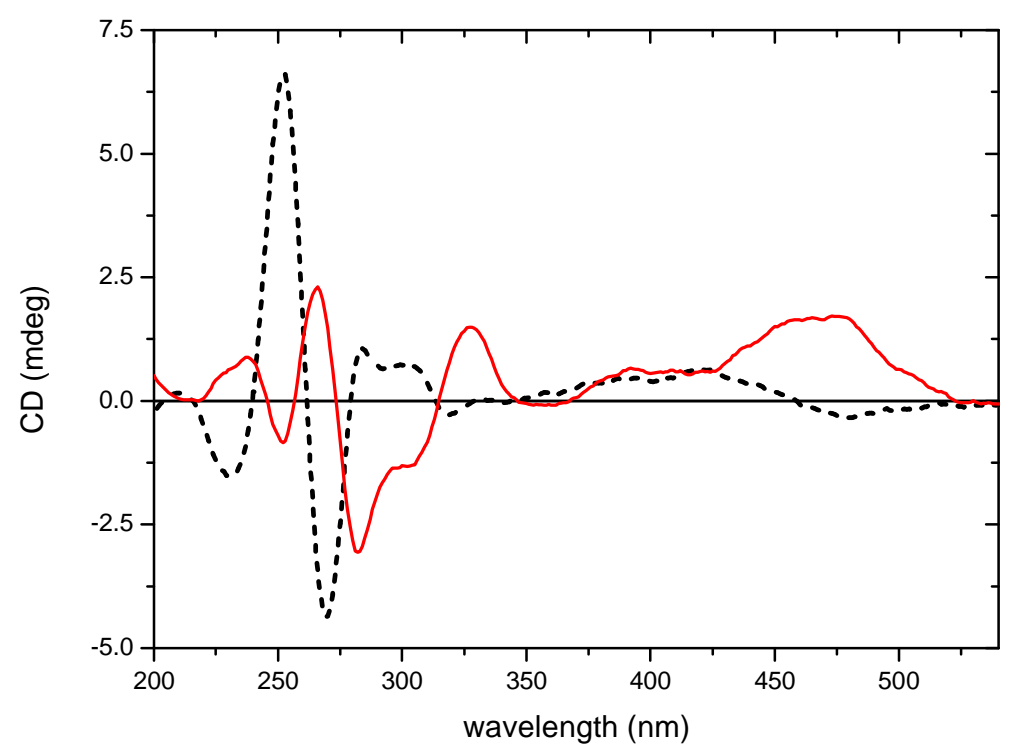

Fig. 5 Perturbation CD spectra defined as DNA-metal complex CD minus CD of pure complex for: A) AT-DNA with $\Delta \Delta$ (solid red) and with $\Lambda \Lambda$ (dashed black), B) GC-DNA with $\Delta \Delta$ (solid red) and $\Lambda \Lambda$ (dashed black). Measurements were made in $25 \mathrm{mM} \mathrm{NaCl}$. The full spectra of free chiral enantiomers and in presence with DNA without subtraction are shown in Fig S4.

Emission kinetics. In line with the circular dichroism study in free solution, also emission kinetics was measured. As previously reported ${ }^{24}$ the present class of ruthenium complexes, that are known to interact with DNA by threading intercalation, while quenched in aqueous solutions become brightly luminescent upon binding to DNA - the "light switch" effect - proving that a dppz moiety is indeed deeply inserted among the stacked bases making it inaccessible to water. The large difference in luminescence intensity enables sensitive kinetic study of threading intercalation by constantly monitoring the luminescence change at $650 \mathrm{~nm}$. Fig 6 represents the kinetic traces of both enantiomers $\Delta \Delta$ and $\Lambda \Lambda$ when interacting with DNA at $37 \mathrm{C}^{\circ}$. Emission 
gradually increases upon addition of GC oligonucleotide having approximately twice stronger intensity in case of $\Delta \Delta$ enantiomer than $\Lambda \Lambda$. Further addition of poly (dAdT) $)_{2}$ results is strong intensity enhancement. Interestingly, the equilibrium is reached relatively fast for $\Lambda \Lambda$ which is also consistent with our LD and CD results indicating external binding mode since only rapid dissociation from HEG-linked GC can explain the fast subsequent intercalation into poly $(\mathrm{dAdT})_{2}$. By contrast, $\Delta \Delta$ is dissociating very slowly reaching eventually an equilibrium in threading intercalation between GC oligonucleotide and poly $(\mathrm{dAdT})_{2}$ after around 3 hours. This observation is in good agreement with $\mathrm{LD}$ and $\mathrm{CD}$ showing enantioselective threading intercalation of sufficiently long GC DNA stretches.

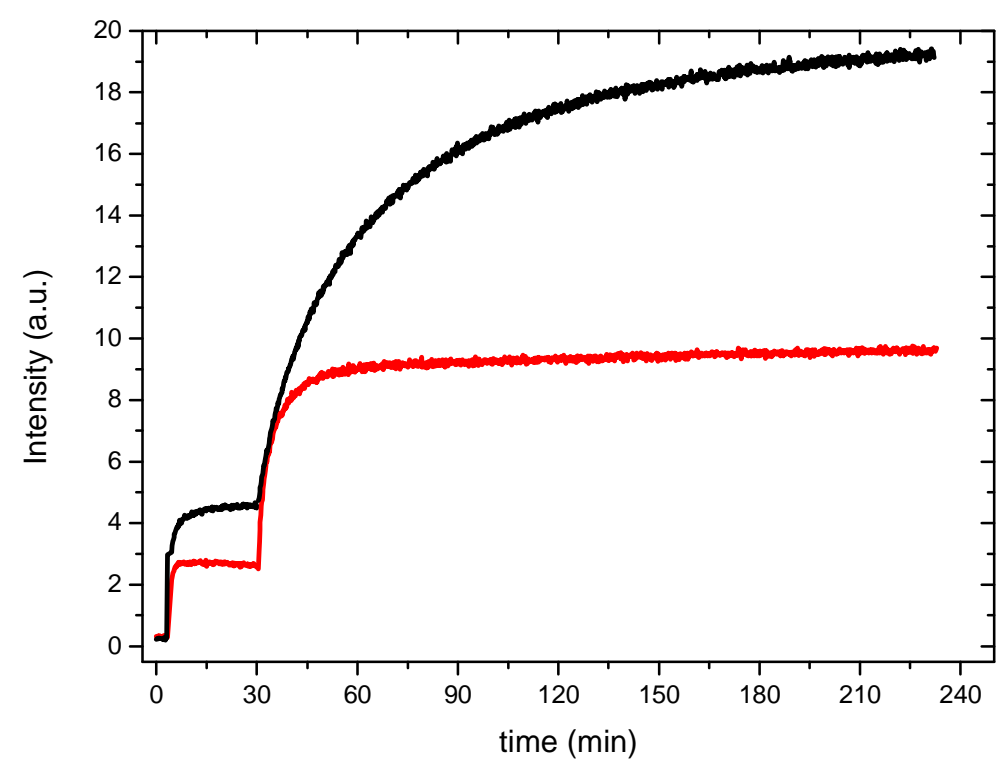

Fig. 6 Kinetics of dissociation for GC-DNA with $\Lambda \Lambda$ (red) or $\Delta \Delta$ (black) measured as change in emission upon addition of extra poly(dAdT) $)_{2}$. All measurement performed at $37 \mathrm{C}^{\circ}$ in $25 \mathrm{mM} \mathrm{NaCl}$ aqueous solutions. 


\section{Discussion}

Previous LD studies of $\Lambda \Lambda$ and $\Delta \Delta\left[\left(11,11^{\prime} \text {-bidppz }\right)(\text { phen })_{4} \mathrm{Ru}_{2}\right]^{4+}$ in presence of long DNA, either mixed sequence (calf thymus DNA) or alternating polynucleotides $[\operatorname{poly}(\mathrm{dAdT})]_{2}$ or $[\operatorname{poly}(\mathrm{dCdG})]_{2}$ aligned in a Couette flow cell, indicated that the binuclear complexes could thread-intercalate into all three DNA contexts ${ }^{19}$. However, for short synthetic HEG-linked oligonucleotides, with sequences having alternating AT base pairs in middle, and flanked by CCGG ends, luminescence studies show that no threading intercalation for any of the enantiomer combinations occurs. In this work we focus on threading intercalation of metal complexes into such HEG linked hairpins of alternating AT and GC oligonucleotides by combining absorbance, CD and emission kinetics, and also LD experiments in the humid PVA system. Distinct hypochromism in the absorption of the local $\pi \rightarrow \pi^{*}$ transitions of dppz, as well as that of the MLCT band, confirms that the binuclear complexes interact closely with nucleobases, as is also supported by CD indicating strong perturbation of DNA structure.

Using these techniques only it is difficult to recognize and distinguish binding mode and position of the complex with respect to DNA helix. However, with the PVA-LD approach we are able to study both the aligned complex itself as well as the DNA-metal complex in PVA. Free metal complex aligns preferentially with its longest dimension parallel with the polymer stretching direction whereas when bound to DNA it orients preferentially perpendicular to stretching direction of polymer matrix. The ruthenium complexes exhibit characteristic absorption spectral shifts upon interaction with DNA which can be also distinguished in their LD spectra. We conclude that the bidppz bridging ligand, which is responsible for the intercalation, as expected is parallel with the plane of the DNA bases [Fig. 3]. Decreasing the relative humidity 
in the closed chamber, and thus the water activity inside the film, does not appear to affect the bidppz band indicating that the DNA-drug complex is stable in the PVA despite lower dielectric constant of the surrounding. Thus we can conclude that threading intercalation indeed occurs in HEG-linked hairpin oligonucleotides composed of repeating AT sequences.

Secondly, a 22bp HEG-linked GC-DNA oligonucleotide was studied: here both enantiomers of the binuclear complex exhibit distinct hypochromism in the dppz and MLCT absorption bands which indicates strong binding, possibly intercalation. However, the CD experiments with the $\Lambda \Lambda$ enantiomer reveals only minor changes in the DNA region suggesting relatively small impact of the complex on overall DNA secondary structure and minor perturbation of the double helix. By contrast, for the $\Delta \Delta$ enantiomer the CD was different indicating stronger influence on DNA structure by the metal complex chromophore. The DNA band is strongly affected by the drug binding consistent with the occurrence of thread-intercalation. In order to confirm the binding mode, we exploited the advantage of the PVA-LD system and compared the LD spectra in presence of DNA for the enantiomers: indeed in presence of a $22 \mathrm{bp} \mathrm{HEG-linked} \mathrm{alternating}$ GC sequence only one stereoisomer, $\Delta \Delta$, is intercalating by threading while the other one, $\Lambda \Lambda$, is virtually externally bound to the double helix. Repeating the experiments in the same way as with AT at varying relative humidity, we note that the external binding mode of $\Lambda \Lambda$ is very sensitive to the water content in polymer matrix. By contrast, the thread-intercalation of $\Delta \Delta$ remains robust both at low as well as high humidity. Changing the relative humidity affects the dielectric constant of PVA. The lower the hydration level is, the lower is the dielectric constant: this will decrease hydrophobic interactions and thereby decrease the binding affinity of the hydrophobic metal complexes for DNA. However, in case of tetravalent intercalators 
electrostatic interactions are more important and one could then expect the opposite phenomenon: lower dielectric constant would give less electrostatic shielding and, hence, stronger binding to DNA. Exactly this behavior is what we conclude in the $\Lambda \Lambda$ case where increase of relative humidity, and thus increase of dielectric constant, leads to destabilization of the DNA-drug complex. This is a behavior opposite to that observed for mono- and di-valent cations where the predominant hydrophobic interactions with PVA annihilates the binding to DNA and results in orientation parallel with the PVA chains even in case of hydration in $100 \%$ r.h.

Another interesting feature of the PVA system is the possibility to monitor dissociation of metal complex from DNA from the gradually changing shape and amplitude of the LD spectrum, from negative (intercalation) to eventually positive as the ruthenium complex instead aligns itself parallel with the PVA chains. Thus the $\Lambda \Lambda$ enantiomer is observed to rearrange upon increasing the water content in film: each LD spectrum can be reproduced as a linear combination of the extreme LD spectra showing a two-state behavior with the ruthenium complex either bound to DNA or free. The $\pi \rightarrow \pi^{*}$ transitions are shifting towards shorter wavelength and LD becoming more positive and finally getting the shape of free $\Lambda \Lambda$ when aligned in PVA [Fig. S3]. At the competition for binding between PVA and DNA the water activity is obviously a key factor affecting the intermolecular interactions. Binding affinity to DNA is stronger in dehydrated conditions and a larger fraction of the metal complex is then interacting with DNA [Fig. 3B]. But when more water molecules are introduced into the system by increasing the relative humidity in the closed humidity chamber, the affinity for DNA becomes weaker and $\Lambda \Lambda$ starts to dissociate. According to a linear combination fit to reproduce the shape of the LD spectrum, approximately 
$25 \%$ of the metal complex is free and aligned with PVA chains and $75 \%$ either still groove bound or bound externally to the DNA.

The concluded specific interaction of the binuclear complex with short GC sequences environment is surprising because it has been previously found in bulk water solution that ruthenium complexes with GC-DNA exhibit usually relatively low quantum yield which was interpreted in terms of groove binding as access of water would there provide strong quenching of the emission. However, that conclusion appears to be only in part justified, because although the emission of threaded $\Delta \Delta$ into GC is indeed relatively weak compared to the quantum yield in poly $(\mathrm{dAdT})_{2}$ it is not negligible (around $\sim 20 \%$ ). According to our results threading is indeed the final binding mode also in short GC-DNA if the sequence is long enough ${ }^{33}$. NMR results indicate that as short as 6 bp oligonucleotides do not become intercalated at all by the ruthenium complex (Lisha $\mathrm{Wu}$ et al. unpublished results). The efficient quenching of the emission of the binuclear ruthenium complex, despite the thread-intercalation, is possibly due to access of water molecules from the DNA grooves when the complex distorts the structure of GC-DNA. Unfortunately, calculating the exact binding angles of the absorbing transition dipole moments with respect to DNA helix is not possible because of overlapping absorption of the monomeric units that may have different orientations. The water quenching mechanism was previously proposed also for the monomer enantiomers $\Lambda$ and $\Delta$ structures, known as efficient intercalators ${ }^{34}$. The dimer complex can be assumed to follow the behavior of the monomer compound.

To sum up, threading intercalation is a final binding mode of $\Delta \Delta$ and $\Lambda \Lambda$ enantiomers upon interaction with AT HEG-linked hairpin oligonucleotides. Surprisingly, with GC-DNA we find that only $\Delta \Delta$ is thread-intercalated whereas the opposite enantiomer $\Lambda \Lambda$ is externally bound. 
Another interesting observation is that intercalation of the binuclear complex is associated with an increase of orientation factor (S) of DNA as compared to free hairpin oligonucleotide aligned in PVA [Fig. S1]. One possible explanation is that the ruthenium complex once threaded between DNA bases is stabilizing the stacking of neighboring bases by neutralizing the repulsion between phosphate groups, thereby decreasing any wobbling or fraying dynamics that may be caused by the decreased dielectric constant of the surrounding. The ruthenium complex may act like a staple at the position where it is threading and, together with HEG at the end of the helix, prevent the strands of duplex from separating transiently, thus somehow zipping up the duplex. Although yet only a speculation such an electrostatically "zipped" DNA would be consistent with the current conclusion of DNA-ruthenium complex being more stable at low humidity while free oligonucleotides be vulnerable to variation of water activity in PVA and changes in hydration shell around DNA.

\section{Conclusions}

We here present for the first time LD results for synthetic short oligonucleotides containing a strongly bound transition metal complex. By combining absorbance, LD, CD and emission spectroscopy we can compare interactions of short hairpin sequences composed of alternating AT or GC with binuclear $\left.\left[11,11^{\prime} \text {-bidppz)(phen }\right)_{4} \mathrm{Ru}_{2}\right]^{4+}$. A significant discovery is the finding of an enantioselective strong threading intercalation into GC-DNA displayed by $\Delta \Delta$, contrasted by a weaker non-intercalative binding of $\Lambda \Lambda$. The binding is virtually very sensitive to changes in dielectric constant of the surrounding environment. A small increase of dielectric constant leads to fast dissociation from thread-intercalation and results in external binding to DNA. To our best 
knowledge this is the first example of a transition metal compound that displays efficient enantioselectivity and exhibits two different binding modes with respect to GC-DNA.

Supporting Information contains 1. LD spectra and calculated $\mathrm{LD}^{\mathrm{r}}$ values of free short oligonucleotides in PVA, 2. Absorption spectra in PVA of oligonucleotides with ruthenium(II) complexes 3. Linear combination of free $\Lambda \Lambda$ in PVA and $\Lambda \Lambda$ with GC-DNA in 75\% r.h. 4. CD of free $\Delta \Delta$ and $\Lambda \Lambda$ and in mixture with GC-DNA5. LD spectrum of stretched PVA in $75 \%$ and $100 \%$ r.h. This information is available free of charge via the Internet at http://pubs.acs.org.

Acknowledgment: The research was supported by European Research Council (ERC) advanced grant to $\mathrm{BN}$.

(1) Bivar T. App. Spect. Rev. 2012, 47, 272-325.

(2) Jaehnchen J.; Purwanto M.; Weisz K. Biopol. 2005, 79, 335-343.

(3) Barton J.; Olmon E.; Sontz P. Coord. Chem. Rev 2011, 255, 619-634.

(4) Bazhulinaa N.; Nikitina A.; Rodina S.; Surovayaa A.; Kravatskya Y.; Pismenskya V.; Archipovaa V; Martin R.; Gurskya G. J. Biomol. Struc. and Dynam. 2009, 26, 701-718.

(5) Norden B.; Rodger A.; Dafforn T. Oxf. Uni. Pr. 2010.

(6) Metcalfe C.; Thomas J. Chem. Soc. Rev. 2003, 32, 215-224.

(7) Kam-Wing Lo K.; Wing-Tat Choi A.; Ho-Tin Law W. Dalt. Trans. 2012, 41, 6021-6047.

(8) Crespy D.; Landfester K.; Schubert U.; Schiller A. Chem. Comm. 2010, 46, 6651-6662.

(9) Bruijnincx P.; Sadler P. Curr. Op. in Chem. Biol. 2008, 12, 197-206

(10) Barton J.; Danishefsky A.; Goldberg J. J. Am. Chem. Soc. 1984, 106, 20172.

(11) Mei H.; Barton J. PNAS 1988, 85, 1339-1343.

(12) Hiort C.; Lincoln P.; Norden B. J. Am. Chem. Soc. 1993, 115, 3448-3454

(13) Olofsson J.; Wilhelmsson M.; Lincoln P. J. Am. Chem. Soc. 2004, 126, 15458-15465 960-4962.

(14) Friedman A.; Chambron J.; Sauvage J.; Turro N.; Barton J. J. Am. Chem. Soc. 1990, 112,

(15) Olson E.; Hu D.; Hormann A.; Jonkman A.; Arkin M.; Stemp E.; Barton J.; Barbara P. J. Am. Chem. Soc. 1997, 119, 11458-11467.

(16) Jenkins Y.; Friedman A.; Turro N.; Barton J. Biochem. 1992, 31, 10809-10816. 
(17) Wilhelmsson M.; Westerlund F.; Lincoln P.; Norden B. J. Am. Chem. Soc. 2002, 124, 12092-12093.

(18) Lincoln P.; Norden B. Chem. Comm. 1996, 18, 2145-2146.

(19) Wilhelmsson M.; Esbjorner E.; Westerlund F.; Norden B.; Lincoln P. J. Phys. Chem. B 2003, 107, 11784-11793. 11554

(20) Novakova O.; Vrana H.; Rodger A.; Sadler P.; Brabec V. Biochem. 2003, 42, $11544-$

(21) Wheate N.; Brodie C.; Collins J.; Kemp S.; Aldrich-Wright J. Mi. Rev. Med. Chem. 2007, $6,627-648$

(22) Zhang J.; Zhang F.; Li H.; Liu C.; Xia J.; Ma L.; Chu W.; Zhang Z.; Chen C.; Li S.; Wang S. Curr. Med. Chem. 2012, 19, 2957-2975.

(23) Westerlund F.; Nordell P.; Blechinger J.; Santos T.; Norden B.; Lincoln P. J. Phys. Chem. B 2008, 112, 6688-6694.

(24) Nordell P.; Westerlund F.; Wilhelmsson M.; Norden N.; Lincoln P. Angew. Chem. Int. Ed. 2007, 46, $2203-2206$.

(25) Nordell P.; Westerlund F.; Reymer A.; El-Sagheer A.; Brown T.; Norden B.; Lincoln P. J. Am. Chem. Soc. 2008, 130, 14651-14658.

(26) Hanczyc P.; Norden B.; Samoc M. Dalt. Trans. 2012, 41, 3123-3125.

(27) Hanczyc P.; Akerman B.; Norden B. Langmuir 2012, 28, 6662-6669.

(28) Matsuoka Y.; Norden B. Biopol. 1983, 22, 1731-1746

(29) Hanczyc P.; Norden B.; Akerman B. J. Phys. Chem. B 2011, 115, 12192-12201.

(30) Norden B. Chem. Phys. 1980, 9, 5032-5038.

(31) Matsuoka Y.; Norden N. Biopol. 1982, 21, 2433-2452.

(32) Lyng R.; Rodger A.; Norden B. Biopol. 1991, 31, 1709-1720.

(33) Nordell P.; Lincoln P. J. Am. Chem. Soc. 2005, 127, 9670-9671.

(34) Lincoln P.; Broo A.; Norden B. J. Am. Chem. Soc. 1996, 118, 2644-2653.

TOC
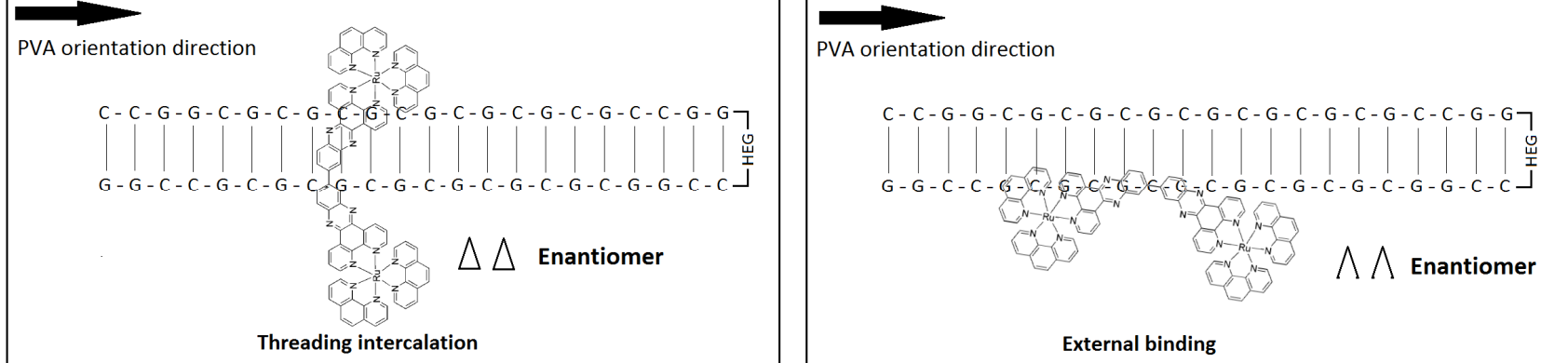